\title{
The prevalence of obesity documen- tation in Primary Care Electronic Medical Records
}

\section{Are we acknowledging the problem?}

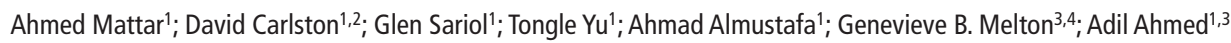

${ }^{1}$ North Central Texas Medical Foundation, Wichita Falls Family Medicine Residency Program, Wichita Falls, Texas, USA;

${ }^{2}$ Department of Psychology, Midwestern State University, Wichita Falls, Texas, USA;

${ }^{3}$ Institute for Health Informatics, University of Minnesota, Minneapolis, Minnesota, USA;

${ }^{4}$ Department of Surgery, University of Minnesota, Minneapolis, Minnesota, USA

\section{Keywords}

Primary care, obesity prevalence, body mass index, electronic medical records documentation, service utilization

\section{Summary}

Background: Although obesity is a growing problem, primary care physicians often inadequately address it. The objective of this study is to examine the prevalence of obesity documentation in the patient's problem list for patients with eligible body mass indexes (BMI) as contained in the patients' electronic medical record (EMR). Additionally, we examined the prevalence of selected chronic conditions across BMI levels.

Method: This study is a retrospective study using EMR data for adult patients visiting an outpatient clinic between June 2012 and June 2015. International Classification of Diseases, Ninth Revision, (ICD-9) codes were used to identify obesity documentation in the EMR problem list. Univariate and multivariate logistic regression analyses were used.

Results: Out of 10,540, a total of 3,868 patients were included in the study. 2,003 (52\%) patients met the criteria for obesity (BMI $\geq 30.0)$; however, only $112(5.6 \%)$ patient records included obesity in the problem list. Moreover, in a multivariate analysis, in addition to age and gender, morbid obesity and cumulative number of comorbidities were significantly associated with obesity documentation, $\mathrm{OR}=1.6$ and $\mathrm{OR}=1.3$, respectively, with $95 \% \mathrm{Cl}[1.4,1.9]$ and $[1.0,1.7]$, respectively. For those with obesity documentation, exercise counseling was provided more often than diet counselling.

Conclusion: Based on EHR documentation, obesity is under coded and generally not identified as a significant problem in primary care. Physicians are more likely to document obesity in the patient record for those with higher BMI scores who are morbidly obese. Moreover, physicians more frequently provide exercise than diet counseling for the documented obese. 


\section{Correspondence to:}

Adil Ahmed

1301 Third Street, Suite 200

Wichita Falls, TX 76301

E-Mail: doola2002@gmail.com

Phone: 507-269-1799
Appl Clin Inform 2017; 8: 67-79

https://doi.org/10.4338/ACI-2016-07-RA-0115

received: July 18, 2016

accepted: November 22, 2016

published: January 25, 2017

Citation: Mattar A, Carlston D, Sariol G, Yu T, Almustafa A, Melton GB, Ahmed A. The prevalence of obesity documentation in primary care electronic medical records: Are we acknowledging the problem? Appl Clin Inform 2017; 8: 67-79

https://doi.org/10.4338/ACl-2016-07-RA-0115

Funding

Research reported in this publication was supported by the National Center for Advancing Translational Sciences (CTSI) of the National Institutes of Health Award Number UL1TR000114. The content is solely the responsibility of the authors and does not necessarily represent the official views of the National Institutes of Health. 


\section{Background}

The Centers for Disease Control and Prevention (CDC) and other sources use an individual's body mass index (BMI) to identify and categorize obesity. For adults, a BMI between 30-39.9 is considered obese and a BMI over 40 is considered morbidly obese [1]. Using this classification system, more than one third of Americans are currently obese [2]. Today's obesity rate is significantly higher than the $13.4 \%$ prevalence rate identified in the late 1970's and early 1980's [3]. Given this increase, obesity is evolving as a healthcare problem in the United States and worldwide [4-7].

The impact of increasing obesity rates is significant. Currently, obesity is one of the greatest drivers of preventable chronic diseases and healthcare costs in the United States. Obese individuals are more than twice as likely to be prescribed medications to manage their comorbid medical conditions compared to their healthy-weight counterparts [8]. Moreover, obesity plays a major role in modifying treatment outcomes associated with comorbid chronic disease [9]. As a result, obesity is associated with significant health care costs, with estimates ranging from $\$ 147$ billion to nearly $\$ 210$ billion annually [10].

Numerous guidelines and recommendations are available regarding the treatment of obesity [2, 11], however, publication of these guidelines has had little impact on the prevalence of obesity. Studies have found that although empirically supported interventions have been identified, frontline providers and clinicians do not routinely incorporate these guidelines into clinical practice [12]. In fact, a recent epidemiological study of weight counseling in primary care researcher found that most primary care physicians (58\%) performed no weight loss counseling for any of their obese patients [12]. Although there are many potential explanations for this dearth of treatment, one parsimonious explanation emerging in the literature is that physicians may not recognize the presence of obesity and/or may not consider obesity to be a significant medical problem at the individual level [13-15].

The ability to identify and manage the care of patients who meet criteria for obesity in ambulatory settings has significantly improved with the increased use of health information technology, especially electronic medical records (EMR) [16]. For example, researchers have found that electronic prompts regarding BMI not only improved the rates of obesity documentation but, more importantly, increased the frequency of patient visits and the rate of weight loss counseling [15,17]. Unfortunately, these studies also suggest that obesity documentation rates still remain low [13-15]. Moreover, a recent systematic review highlighted that few studies have examined if the EHR provided clinicians the tools to screen and address patients who were overweight and obese [18].

The primary aim of this study is to examine the prevalence of obesity documentation in the patient's problem list in the EMR and the impact of documentation on patient care. Additionally, we examined the prevalence of selected major chronic obesity related conditions (i.e., diabetes, hypertension, coronary heart disease, depression, chronic obstructive pulmonary disease) across different BMI levels. Part of this work was presented at the International Congress on Obesity (ICO) in Vancouver, Canada, May 2016.

\section{Methods}

We conducted a retrospective review of adult patients visiting an outpatient clinic between June 2012 and June 2015. The institutional review board of Midwestern State University approved the study (Institutional Review Board \#15102701).

\section{Study Setting and Populations}

We reviewed patient EMR gathered through routine care at the Wichita Falls Family Medicine Clinic. The clinic is host for a private family medicine residency program with five faculty members and 24 residents spanning three post-graduate levels. The clinic is located in the city of Wichita Falls (population approximately 104,553), the center of Wichita County and a population center of North Texas.

The clinic uses e-MD ${ }^{\odot}$ as its EMR system. This system was introduced to the clinic in 2009. e-MD is a certified EMR that includes appointment management, clinical notes, labs, and billing, among 
other features. For each visit, a nurse charts the primary reason for the patient's visit in addition to manually entering the patient's vital signs, including the patient's height and weight. A BMI score is automatically calculated by the EMR and presented in the patient's chart as part of the vital signs section.

\section{Data Extraction}

The EMR saves all data on a local secure server, using the Microsoft SQL ${ }^{\odot}$ database. Data stored includes patient demographics, front-desk registration information, clinical charting information, vital signs, laboratory results, medications proscribed, e-prescribing system order data, and other revenue cycle/administrative data, including associated International Classification of Diseases, Ninth Revision, (ICD-9) codes which were used to create the patient's problem list. The Microsoft SQL Server 2008 R2 Management Studio ${ }^{\odot}$ was used to extract data from the associated EHR database for the current project. We used ICD-9 code 278 to identify obese patients seen between January 2012 and June 2015, only two codes were identified in our database (278.01,278.02). Adults aged 18 years and older with two or more visits during the study window were included in the study. Children and pregnant women were excluded, because strict BMI interpretation is particularly less meaningful in these patient populations.

Data for diet and exercise counseling were not entered in a structured format into the EMR, but were most often documented as free text within the assessment and plan sections of the chart. For feasibility, data regarding exercise and dietary counseling were manually obtained from those with obesity documented in the problem list 115 (100\%) and from a randomly selected comparison group of those meeting obesity criteria without obesity documented in the problem list214 (15\%). All manual extraction was done by two independent investigators (blinded to each other's results) using CDC definition of obesity $(\mathrm{BMI} \geq 30)$. All differences were resolved by a third blind reviewer. This procedure yielded a good inter-observer agreement, $\kappa=0.7,95 \%$ CI [0.5-0.8]. Selected comorbidities were also extracted using their appropriate ICD -9 codes.

\section{Statistical Approach}

Our primary outcome was the percentage of patients with obesity documentation, out of total obesity eligible BMI. Additionally, the characteristics of those with and without obesity documentation were a secondary outcome. Finally, our third outcome was the prevalence of counselling provided across the two groups. The baseline characteristics data are presented as totals and percentages for categorical variables and median interquartile range (IQR) for continuous variables. These data were compared using Wilcoxon rank-sum and Fisher's exact tests, respectively. For those with $\mathrm{BMI} \geq 30$, multivariate logistic regression was used to identify the independent variables associated with obesity documentation.

Three statistical models were run for sensitivity analysis. All models included the baseline characteristics as covariates, including age, gender, race, and type of insurance, in addition to BMI-related variables. In Model 1, we included BMI as a continuous variable; in Model 2, we included morbid obesity status in addition to BMI; and in Model 3, we included BMI, morbid obesity status $(\mathrm{BMI} \geq 40)$, and the total number of comorbidities as covariates. Odd ratios with $95 \%$ confidence intervals were calculated for each variable in the model.

For those with obesity documentation, the first visit during the study window was used as time zero. Change in BMI over three time intervals (3, 6 and 12 months) was calculated. Additionally, the median number of office visits was calculated using the same intervals. A matched paired analysis was used for the cross group comparisons and the $p$-value was calculated using Wilcoxon Signed Rank test. STATA (Version 14.1) and JMP statistical software $\odot$ (Version 11, SAS Institute) was used for all data analyses. 


\section{Results}

During the study period, patient information for 10,540 medical records was evaluated. Included in this study were 3,868 patients with 15,790 total office visits. The median number of visits for participants was two. Figure 1 illustrates the sample flow chart and associated exclusions.

The prevalence of obesity with EMR-generated BMI, BMI $\geq 30.0$, was 2,003, representing $51.7 \%$ of included patients. Only 112 of the BMI eligible patients (5.6\%) had obesity documented in their patient problem lists. Baseline characteristics for those with and without obesity documentation are presented in $>$ Table 1 . Compared to those without documentation, patients with documented obesity were significantly younger, more likely to be female, had a higher median BMI, and were more frequently morbidly obese.

Physicians provided dietary counseling for $30 \%$ and exercise counseling for $40 \%$ of patients with documented obesity. Dietary counseling rates in a randomly selected comparison group of undocumented patients who meet diagnostic criteria for obesity were similar $(31 \%, p=0.92)$. Whereas, exercise counseling rates were significantly lower in the same group of undocumented patients $(29 \%, p$ $=0.03$ ). Baseline demographic and clinical characteristics did not differ significantly between manually and electrically collected data ( $\$$ Supplemental Table 1$)$.

In the multivariate analysis age and gender were significant in all three models. When morbid obesity was included in the second model, it was also significantly associated with documentation OR $[95 \% \mathrm{CI}]=1.6[1.4-1.9]$. Finally, when the cumulative number of comorbidities was added to the model, it was also significantly associated with documentation of obesity, OR $[95 \% \mathrm{CI}]=1.3[1.0$ -1.7] ( Supplemental Table 2).

Compared to those with $\mathrm{BMI}<30$, the presence of obesity was associated with increased rates of hypertension, $38 \%$ and $62 \%$ respectively, $p<0.01$; diabetes, $30 \%$ and $70 \%$ respectively, $p<.01$; and heart disease, $42 \%$ and $58 \%, p<0.01$. However, compared to those with $\mathrm{BMI}<30$, individuals meeting criteria for obesity were not more likely to be diagnosed with depression, $50 \%$ and $50 \%$ respectively, $p=0.19$, or chronic obstructive pulmonary disease, $46 \%$ and $54 \%$ respectively, $p=0.67$. Analysis of service utilization, defined as the number of obesity specific or obesity related-illness visits, was associated with increased BMI (i.e., BMI <30, BMI 30-39.9, and BMI > 40). Specifically, higher BMI scores were associated with greater numbers of visits for each comorbid condition ( $>$ Figure 2 ).

Among those with documented obesity, service utilization increased with increased BMI level. When measured at 3,6, and >12 month intervals, BMI increased over time with mean differences across the three intervals respectively ( $>$ Figure 3 ).

\section{Discussion}

In the present study, we demonstrated higher than expected prevalence rates of obesity in our clinical sample. The elevated prevalence of obesity in our sample relative to the national average can be explained by the low socioeconomic status of our current sample. Additionally, we demonstrated low prevalence of obesity documentation in medical records. We identified predictors of documentation including age, gender, number of comorbidities and obesity severity. Specifically, we demonstrated that those with morbid obesity were much more likely to have a diagnosis of obesity included in their EHR, possibly due to the visual undeniability of problem severity. We also demonstrated that among those with high BMI, less than half had diet and exercise counseling. However, documentation was associated with a greater probability of exercise counseling. Finally, we also showed that higher BMI categories are associated with greater comorbidity prevalence and with a higher number of visits incurred by selected individual comorbidity.

The obtained obesity documentation rates in our study are consistent with the rates of obesity documentation previously reported using hospitalized patients. For example, Azhdam et al. found that less than 1 percent $(<1 \%)$ of hospitalized obese and overweight patients had any documentation of obesity included in their discharge summary [19]. Furthermore, the authors noted that only 13.2\% had their weight status noted anywhere in their medical record [19]. Other researchers reported similarly low rates of documentation with only $1.7 \%$ of hospitalized obese patients having the diagnosis at discharge[20]. 
Low documentation rates have also been found in outpatient samples $[14,15]$. For example, both Banerjee et al. and Bordowitz et al. found that less than one third of outpatients carried an obesity diagnosis in their medical records. However, it should be noted that the obtained documentation rates in our study were significantly lower than those obtained in previous similar studies. Although there is no testable explanation, the data collection sites differed on three significant variables. Specifically, previous research was conducted in university clinics in states with relatively low obesity population rates and with a diverse patient economic background; whereas, the current sample was collected in a non-university based clinic in a state with relatively high obesity prevalence and included, primarily, a low income sample[21].

Multiple barriers have been identified in previous studies that could have contributed to the lack of addressing obesity as a separate medical condition rather than a sequela. Patients may have been deterred from discussing their obesity with their primary care physician because of their ambivalence about the treatment options and stigmatizing emotional state associated with an obesity diagnosis [22]. On the other hand, some reports suggest that the lack of knowledge and familiarity with guidelines is another reason for physicians to shy away from addressing the problem [23]. A recent study suggested that more educational programs are needed to improve physician knowledge and competency in treating patients with obesity [24]. This body of research also shows that education is associated with the delivery of higher quality counseling [25].

Consistent with previous research, we also found that documentation of obesity as a medical problem was associated with greater physician attention to patient weight, specifically an increased prevalence of exercise counseling [22]. This finding supports Banerjee et al's assertion that inclusion of an obesity diagnosis could lead to significant improvement in physician attention to patient weight [15]. Interestingly, in our study, the rate of diet counselling was similar across documentation groups; however, exercise counseling rates were significantly higher in the documented group. Although some have shown that the documentation of obesity in medical records indicates that physicians pay more attention and more often address obesity in clinical settings [15], in the present study, only a third of primary care visits included counseling for diet, and exercise counseling rates were also relatively low. Although it was not feasible to examine the time allocated for counseling in our study, other studies have shown that counseling time is minimal, with one study showing only 1.75 minutes (8.0\%) of total office visit time was related to overweight and obesity counseling [26].

The US Preventive Services Task Force set guidelines for primary care clinics and recommended that screening for obesity using BMI should be done for all adults. Similarly, the American Academy of Family Physicians has recommended that intensive counseling and behavioral interventions should be offered to adults diagnosed with obesity [27]. The most recent recommendations in 2015 from the American College of Cardiology/American Heart Association and The Obesity Society, introduced five major areas for obesity management in adults, starting with identifying those at risk, physician counseling, and guidelines for treatment with diet, lifestyle intervention, and surgery [28]. These new recommendations serve as a roadmap for providing primary care and family physicians with the current best practices to be incorporated into practice [29]. One simple, initial step to increasing guideline compliance may be to encourage physicians to document obesity as a medical problem in the patient's EMR.

The same clinical recommendations promoted intensive behavioral intervention aimed at lifestyle modification. This intervention, characterized by a minimum of 14 visits within a six-month period, has been found to be effective [30]. Our group is presently studying the feasibility and efficacy of delivering physician-supervised, technology-driven intensive behavioral therapy for low socioeconomic status patients similar to the ones in this study.

Although administrative data like ICD-9 codes lack the accuracy and reliability, our regress validation with good interobserver agreement improves the reliability of our results. However, our study has several limitations. First, the retrospective nature of our study limits our ability to infer causal effects and limits our analyses to associations. Second, we used the problem list as a surrogate for addressing the problem as part of the clinical encounter. It is possibly that there could have been counseling related to the problem that was verbally provided but not documented within the medical records. Although this may have been the case in our study, it would not explain the low prevalence in obesity documentation in our study. Third, although defining obesity by patient BMI has been validated and is widely accepted at both the individual and population level, it has some limitations. 
Chiefly, BMI may not reflect the actual body fat component particularly for those in the overweight category [31]. Finally, the fact that this was a single-center study that used one EHR system limits the generalizability to similar settings and populations.

In conclusion, these findings, together with the existing literature, suggest that obesity is generally not recognized as a primary medical problem. As a result, counseling is provided infrequently, perhaps because of the lack of structural approach to the problem. Additionally, obesity is associated with an increase in the prevalence of comorbid conditions and associated service utilization. However, higher numbers of visits might not positively impact patient BMIs. Continued research is needed to identify effective methods for physician counseling and behavioral therapy approaches in the treatment of obesity.

\section{Clinical Relevance Statement}

Obesity is not recognized frequently in primary care setting. Physicians counselled those with significantly higher BMI which reflect in the documentation in the problem list.

\section{Conflict of Interest Statement}

The authors declare that they have no conflicts of interest in relation to the presented study.

\section{Human Subjects Protects}

Humans subjects were not directly included in the project, institutional review board approved the study.

\section{Institution}

This work was performed at Family Health Center Clinic Operated by Wichita Falls Family Practice Residency Program. Wichita Falls, TX

\section{Presentation}

The abstract of this work was presented at the International Congress on Obesity (ICO) in Vancouver, Canada, in May 2016 


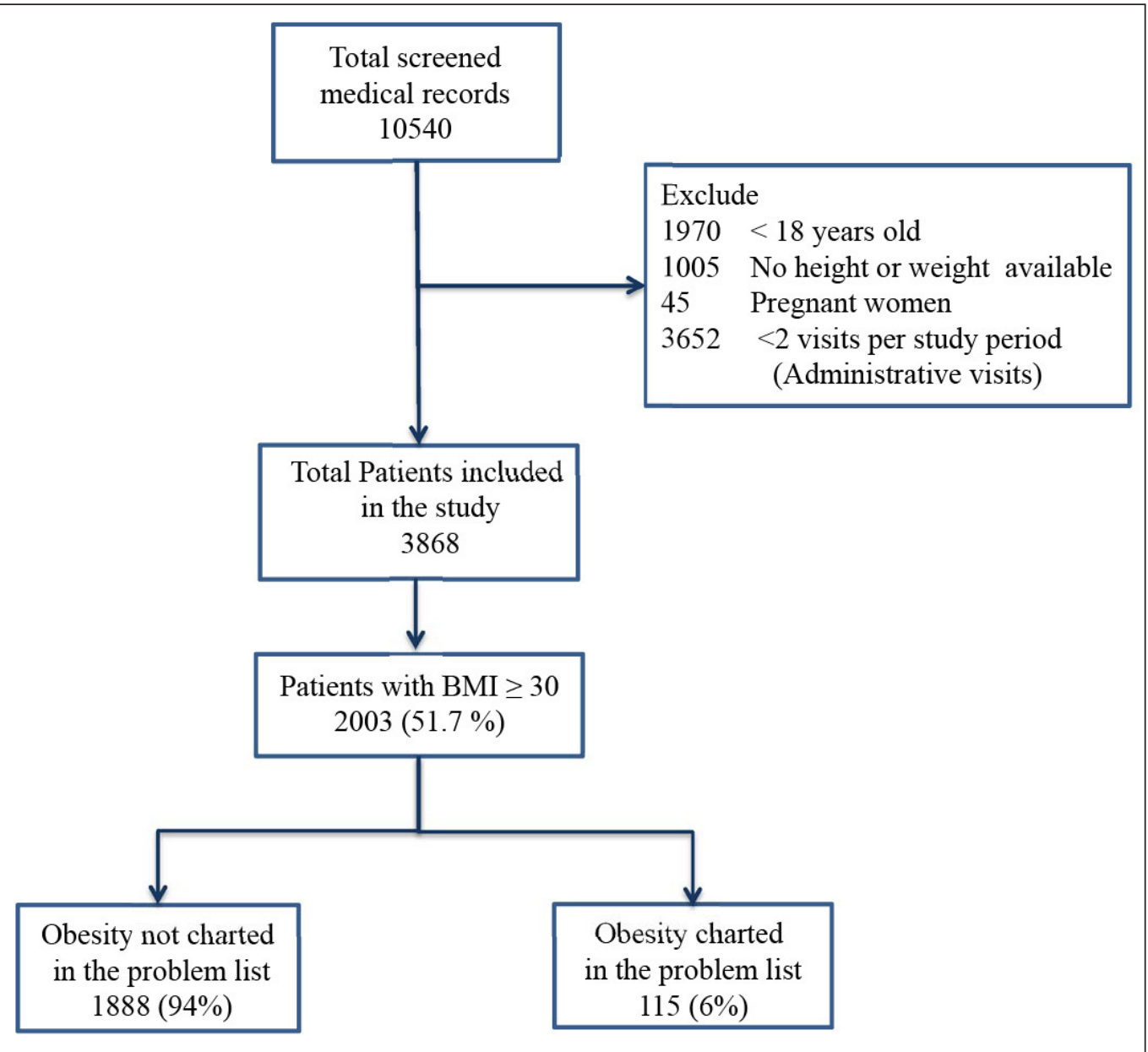

Fig. 1 Patient Flow Chart. Included patients were seen in an outpatient family practice clinic between June 2012 and June 2015. Most patients with fewer than two visits during the study window were seen as walk-in patients for acute problems, for sport physicals, or disability evaluations. These patients were excluded, as they were patients for whom continuity of care was not provided. 


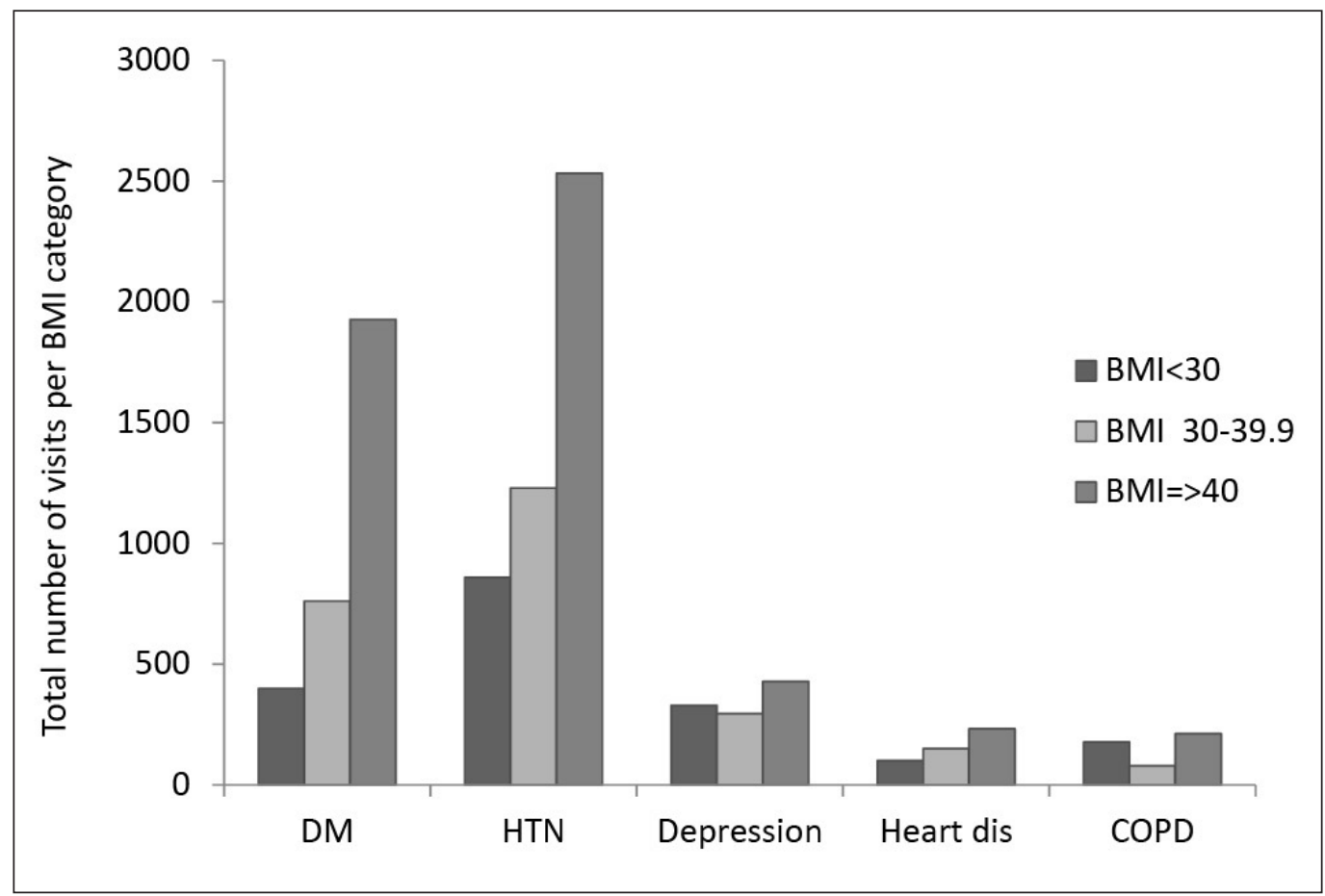

Fig. 2 Patient Encounter Totals across BMI Category for Obesity-related Comorbidities. DM=Diabetes Mellitus, HTN=Hypertension, Heart dis= Heart disease, $C O P D=C h r o n i c$ Obstructive Pulmonary Disease.

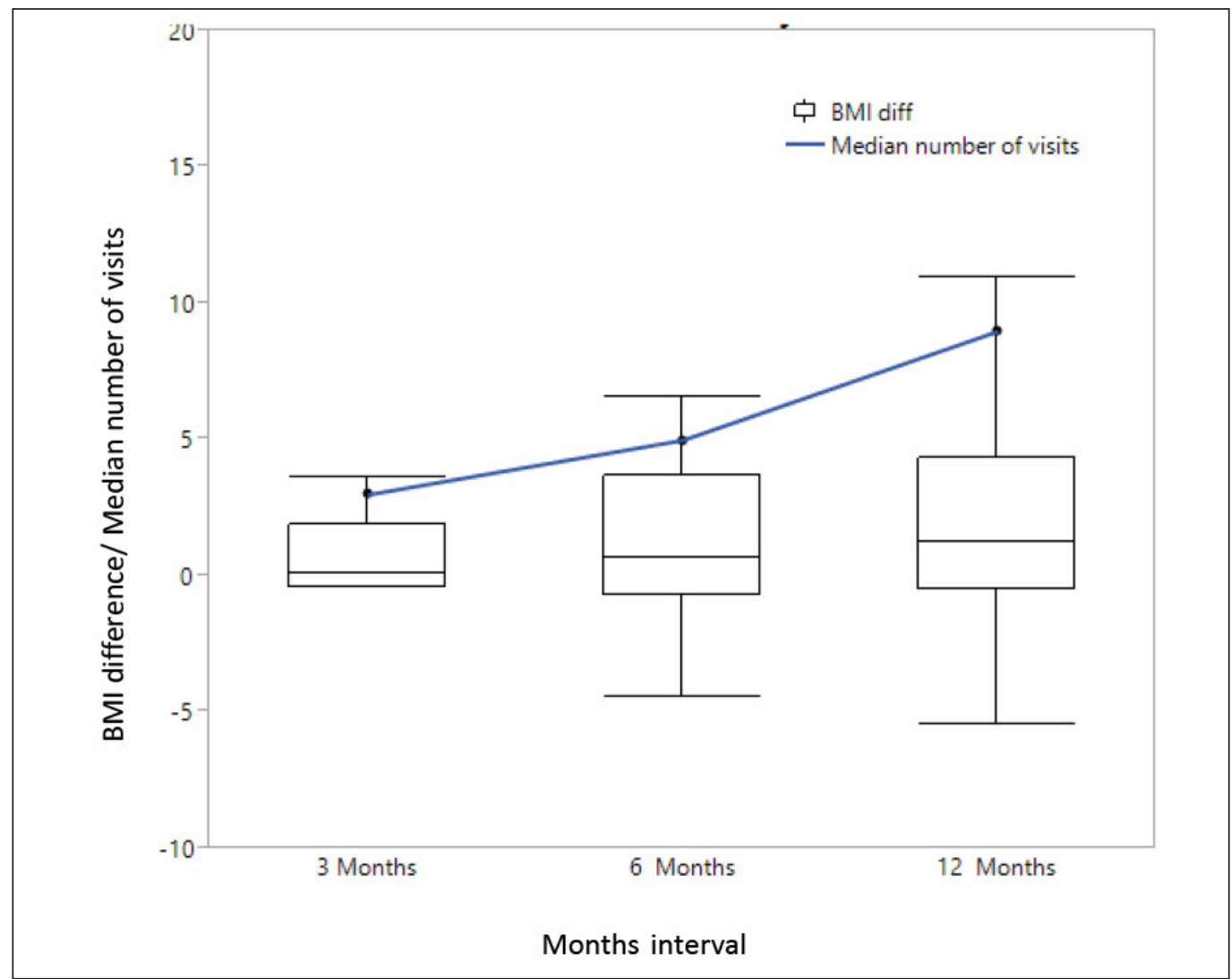

Fig. 3 Change in BMI and Median Number of Visits over Time for Patients with Obesity Documentation. Box plots represent the change in BMI between the initial visit and the 3 month $(n=8), 6$ month $(n=15)$ and $\geq 12$ $(n=93)$ month intervals. The line represents the median number of physician visits across the same visits intervals. 
Table 1 Clinical and Demographic Patient Characteristics across Obesity Documentation Groups

\begin{tabular}{|c|c|c|c|}
\hline & $\begin{array}{l}\text { Undocumented } \\
(n=1,891)\end{array}$ & $\begin{array}{l}\text { Documented } \\
(n=112)\end{array}$ & $p$-value \\
\hline $\mathrm{Age}^{\mathrm{c}}$ & $48.7(36.7,56.2)$ & $44.9(33.55,53.8)$ & $0.01^{d}$ \\
\hline \multicolumn{4}{|l|}{ Gender } \\
\hline Female & $1,113(58.9 \%)$ & $84(75.0 \%)$ & \multirow[t]{2}{*}{$<0.01^{e}$} \\
\hline Male & $778(41.1 \%)$ & $28(25.0 \%)$ & \\
\hline \multicolumn{4}{|l|}{ Ethnicity } \\
\hline Hispanic or Latino & $105(5.6 \%)$ & $7(6.3 \%)$ & \multirow[t]{2}{*}{$0.68^{e}$} \\
\hline Not Hispanic or Latino & $1,774(94.3 \%)$ & $105(93.8 \%)$ & \\
\hline \multicolumn{4}{|l|}{ Race } \\
\hline White & $1,429(76.1 \%)$ & $88(78.6 \%)$ & \multirow[t]{3}{*}{$0.92^{\mathrm{e}}$} \\
\hline African American/Black & $352(18.8 \%)$ & $19(17.0 \%)$ & \\
\hline Other & $96(5.1 \%)$ & $5(4.5 \%)$ & \\
\hline \multicolumn{4}{|l|}{ Insurance } \\
\hline County Indigent ${ }^{f}$ & $258(27.3 \%)$ & $18(40.9 \%)$ & \multirow[t]{5}{*}{$0.08^{e}$} \\
\hline Medicaid & $289(30.5 \%)$ & $12(27.3 \%)$ & \\
\hline Medicare & $216(22.8 \%)$ & $9(20.5 \%)$ & \\
\hline Private Insurance & $94(9.9 \%)$ & $5(11.4 \%)$ & \\
\hline Self-pay & $89(9.4 \%)$ & $0(0.0 \%)$ & \\
\hline Body Mass Index & $34.6(31.6,39.5)$ & $42.6(36.05,54.05)$ & $<0.01^{d}$ \\
\hline Morbid Obesityg & $507(26.8 \%)$ & $74(66.1 \%)$ & $0.53^{e}$ \\
\hline Number of comorbidities ${ }^{c}$ & $1(1,2)$ & $1(0,1)$ & $<.01 \mathrm{e}$ \\
\hline
\end{tabular}

Note. andocumented obesity patients met diagnostic criteria for obesity based upon their BMI score but do not have obesity included in their corresponding problem lists. ${ }^{b}$ Documented obesity patients both met diagnostic criteria for obesity based upon their BMI score and had obesity included in their corresponding problem lists. 'Data

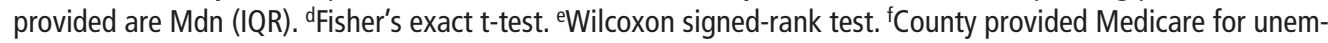
ployed and uninsured. ${ }^{\mathrm{B} B M \mathrm{I}}>40$ 


\section{References}

1. Ogden CL, et al. Prevalence of obesity and trends in body mass index among US children and adolescents, 1999-2010. JAMA 2012; 307(5): 483-490.

2. Yang L, Colditz GA. Prevalence of Overweight and Obesity in the United States, 2007-2012. JAMA Intern Med 2015; 175(8): 1412-1413.

3. Flegal KM, Carroll MD, Kuczmarski RJ, Johnson CL. Overweight and obesity in the United States: prevalence and trends, 1960-1994. Int J Obes Relat Metab Disord 1998; 22(1): 39-47.

4. Novotny R, et al. Systematic Review of Prevalence of Young Child Overweight and Obesity in the United States-Affiliated Pacific Region Compared With the 48 Contiguous States: The Childrens Healthy Living Program. Am J Public Health 2015; 105(1): e22-e35.

5. Singh GK, Lin SC. Dramatic Increases in Obesity and Overweight Prevalence among Asian Subgroups in the United States, 1992-2011. ISRN Prev Med2013;2013:898691.

6. Rahmani A, et al. Investigation of the Prevalence of Obesity in Iran: a Systematic Review and Meta-Analysis Study. Acta Med Iran 2015; 53(10): 596-607.

7. Arbex AK, Rocha DR, Aizenberg M, Ciruzzi MS. Obesity epidemic in Brazil and Argentina: a public health concern. J Health Popul Nutr2014; 32(2): 327-334.

8. Teuner CM, et al. Impact of BMI and BMI change on future drug expenditures in adults: results from the MONICA/KORA cohort study. BMC health services research 2013; 13: 424.

9. Li Q, Blume SW, Huang JC, Hammer M, Ganz ML. Prevalence and healthcare costs of obesity-related comorbidities: evidence from an electronic medical records system in the United States. J Med Econ 2015; Sep 4: 1-9.

10. Cawley J, Meyerhoefer C. The medical care costs of obesity: an instrumental variables approach. J Health Econ 2012; 31(1): 219-230.

11. Pogach LM, et al. Development of evidence-based clinical practice guidelines for diabetes: the Department of Veterans Affairs/Department of Defense guidelines initiative. Diabetes Care 2004; 27 (Suppl. 2): B82-B89.

12. Kraschnewski JL, et al. The epidemiology of weight counseling for adults in the United States: a case of positive deviance. International journal of obesity 2013; 37(5): 751-753.

13.Davis NJ, et al. Obesity management: physician practice patterns and patient preference. Diabetes Educ 2006; 32(4): 557-561.

14. Bordowitz R, et al The use of an electronic medical record to improve documentation and treatment of obesity. Fam Med2007 Apr;39(4):274-9.

15. Banerjee ES, et al Adding obesity to the problem list increases the rate of providers addressing obesity. Fam Med2013 Oct;45(9):629-33.

16. Marcotte L, et al. Achieving meaningful use of health information technology: a guide for physicians to the EHR incentive programs. Arch Intern Med2012 May 14;172(9):731-6.

17. Banerjee ES, Gambler A, Fogleman C. Adding obesity to the problem list increases the rate of providers addressing obesity. Fam Med 2013; 45(9): 629-633.

18. Baer HJ, et al. Using electronic health records to address overweight and obesity: a systematic review. Am J Prev Med 2013; 45(4): 494-500.

19. Azhdam DB, Reyhan I, Grant-Guimaraes J, Feinstein R. Prevalence and documentation of overweight and obesity in hospitalized children and adolescents. Hosp Pediatr 2014; 4(6): 377-381.

20. Woo JG, Zeller MH, Wilson K, Inge T. Obesity identified by discharge ICD-9 codes underestimates the true prevalence of obesity in hospitalized children. J Pediatr2009; 154(3): 327-331.

21. Ogden CL, Lamb MM, Carroll MD, Flegal KM. Obesity and socioeconomic status in adults: United States, 2005-2008. NCHS Data Brief 2010 Dec(50): 1-8.

22. Brown I, Thompson J, Tod A, Jones G. Primary care support for tackling obesity: a qualitative study of the perceptions of obese patients. Br J Gen Pract 2006; 56(530): 666-672.

23. Forman-Hoffman V, Little A, Wahls T. Barriers to obesity management: a pilot study of primary care clinicians. BMC Fam Pract 2006; 7: 35.

24.Stanford FC, et al. The Role of Obesity Training in Medical School and Residency on Bariatric Surgery Knowledge in Primary Care Physicians. Int J Family Med 2015; 2015: 841249.

25. Jay M, Schlair S, Caldwell R, Kalet A, Sherman S, Gillespie C. From the patient's perspective: the impact of training on resident physician's obesity counseling. J Gen Intern Med 2010; 25(5): 415-422.

26. Tsai AG, Abbo ED, Ogden LG. The time burden of overweight and obesity in primary care. BMC Health Serv Res 2011; 11: 191.

27. Screening for and management of obesity in adults: recommendation statement. American family physician 2012; 86(10): 1-3. 
28. Jensen MD, et al. Guideline for the management of overweight and obesity in adults: a report of the American College of Cardiology/American Heart Association Task Force on Practice Guidelines and The Obesity Society. J Am Coll Cardiol 2014; 63(25 Pt B): 2985-3023.

29. Ryan DH, et al. What the new obesity guidelines will tell us. Curr Opin Endocrinol Diabetes Obes 2013; 20(5): 429-433.

30. Jensen MD, Ryan DH. New obesity guidelines: promise and potential. JAMA 2014; 311(1): 23-24.

31. Romero-Corral A, et al. Accuracy of body mass index in diagnosing obesity in the adult general population. Int J Obes (Lond) 2008; 32(6): 959-966. 
Supplemental Table 1 Sensitivity analysis between randomly selected manually collected data and the electrically extracted data.

\begin{tabular}{|c|c|c|c|c|}
\hline \multicolumn{2}{|l|}{ Variable } & $\begin{array}{l}\text { Random manually collected data } \\
\text { (N 214) }\end{array}$ & $\begin{array}{l}\text { Electronically extracted data } \\
\text { (N 3801) }\end{array}$ & $p$-value \\
\hline \multicolumn{2}{|c|}{ Age (Median (IQR)( } & $50(38,59)$ & $49(35,57)$ & 0.05 \\
\hline \multicolumn{2}{|c|}{ Female gender $n(\%)$} & $129(60)$ & $2460(56)$ & 0.21 \\
\hline \multirow{3}{*}{$\begin{array}{l}\text { Ethnicity } \\
\mathrm{n}(\%)\end{array}$} & Not Hispanic or Latino & $198(92)$ & $3569(93)$ & \multirow[t]{3}{*}{0.35} \\
\hline & Hispanic or Latino & $13(6)$ & $212(5)$ & \\
\hline & Declined & $3(1.4)$ & $20(0.5)$ & \\
\hline \multirow{6}{*}{$\begin{array}{l}\text { Race } \\
\mathrm{n}(\%)\end{array}$} & White & $155(72)$ & $2851(75)$ & \multirow[t]{6}{*}{0.53} \\
\hline & Black or African American & $44(20)$ & $704(18)$ & \\
\hline & American Indian or Alaska Native & $1(0.4)$ & $11(0.3)$ & \\
\hline & Asian & $1(0.5)$ & $45(1.1)$ & \\
\hline & Native Hawaiian or Other Pacific Islander & $1(0.4)$ & $3(0.1)$ & \\
\hline & Declined & $24(0.6)$ & $3(1.4)$ & \\
\hline \multicolumn{2}{|c|}{ BMI (Median (IQR)) } & $35(31,39)$ & $29(25,35)$ & 0.82 \\
\hline
\end{tabular}

Note: Baseline demographic and clinical characteristics did not differ significantly between manually and electrically collected data

Supplemental Table 2 Multivariate analysis of predictors of obesity documentation

\begin{tabular}{|c|c|c|c|}
\hline & $\begin{array}{l}\text { Model } 1 \\
\text { OR }[95 \% \mathrm{CI}] \\
n=1,894\end{array}$ & $\begin{array}{l}\text { Model } 2 \\
\text { OR }[95 \% \mathrm{CI}] \\
n=1,929\end{array}$ & $\begin{array}{l}\text { Model } 3 \\
\text { OR }[95 \% \mathrm{CI}] \\
n=1,898\end{array}$ \\
\hline Age & $0.97[0.95-0.98]$ & $0.98[0.97-0.99]$ & $0.97[0.95-0.98]$ \\
\hline Female & $0.58[0.37-0.91]$ & $0.59[0.37-0.93]$ & $0.58[0.37-0.91]$ \\
\hline White & $1.20[0.71-2.04]$ & $1.10[0.34-3.51]$ & $1.30[0.38-4.46]$ \\
\hline Other races & $0.88[0.306-2.53]$ & $1.02[0.33-3.10]$ & $1.20[0.71-2.04]$ \\
\hline Hispanic & $1.12[0.448-2.80]$ & $1.10[0.41-2.87]$ & $1.08[0.40-2.89]$ \\
\hline Medicaid & $0.94[0.407-2.19]$ & $1.10[0.52-2.32]$ & $0.89[0.36-2.18]$ \\
\hline Medicare & $1.22[0.491-3.05]$ & $0.64[0.24-1.62]$ & $0.98[0.380-2.54]$ \\
\hline Private & $0.63[0.17-2.25]$ & $0.68[0.25-2.54]$ & $0.64[0.13-3.01]$ \\
\hline COPD & $0.57[0.07-4.38]$ & $1.42[0.21-9.28]$ & \\
\hline Diabetes mellitus & $1.46[0.33-6.47]$ & $1.58[0.35-7.17]$ & \\
\hline Depression & $1.38[0.29-6.61]$ & $1.76[0.36-8.61]$ & \\
\hline Hypertension & $1.40[0.32-6.13]$ & $1.45[0.32-6.51]$ & \\
\hline Cardiovascular disease & $1.42[0.22-9.05]$ & $1.11[0.24-5.03]$ & \\
\hline BMI & $1.00[0.99-1.00]$ & $1.00[0.99-1.00]$ & $1.00[0.99-1.00]$ \\
\hline Morbid obesity & & $1.68[1.46-1.93]$ & $1.60[1.39-1.84]$ \\
\hline Number of Comorbidities & & & $1.33[1.03-1.73]$ \\
\hline
\end{tabular}

Note. Multivariate logistic regression, three statistical models were run for sensitivity analysis. All models included the baseline characteristics as covariates, including age, gender, race, and type of insurance, in addition to BMI-related variables. In model 1, we included BMI as a continuous variable; in model 2, we included morbid obesity status $[\mathrm{BMI} \geq 40$ ] in addition to BMl; and in model 3, we included BMI, morbid obesity status, and the total number of comorbidities as covariates. Odd ratio with 95\% confident interval OR [95\%] was calculated for variables in the model. 